MS\&T Division

CRADA Final Report

For

CRADA Number NFE-09-02242

Engine Materials Compatibility with Alternate Fuels

S. Pawel

Oak Ridge National Laboratory

D. Moore USCAR

Prepared by

Oak Ridge National Laboratory Oak Ridge, TN 37831

managed by

UT-BATTELLE, LLC

for the

U.S. Department of Energy

under contract DE-AC05-00OR22725

Approved for

Distribution (tbd)

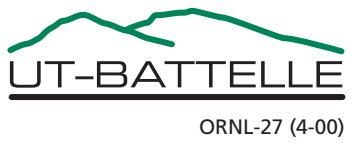




\section{DOCUMENT AVAILABILITY}

Reports produced after January 1, 1996, are generally available free via the U.S. Department of Energy (DOE) Information Bridge.

Web site http://www.osti.gov/bridge

Reports produced before January 1, 1996, may be purchased by members of the public from the following source.

National Technical Information Service

5285 Port Royal Road

Springfield, VA 22161

Telephone 703-605-6000 (1-800-553-6847)

TDD 703-487-4639

Fax 703-605-6900

E-mail info@ntis.fedworld.gov

Web site http://www.ntis.gov/support/ordernowabout.htm

Reports are available to DOE employees, DOE contractors, Energy Technology Data Exchange (ETDE) representatives, and International Nuclear Information System (INIS)

representatives from the following source.

Office of Scientific and Technical Information

P.O. Box 62

Oak Ridge, TN 37831

Telephone 865-576-8401

Fax 865-576-5728

E-mail reports@adonis.osti.gov

Web site http://www.osti.gov/contact.html

This report was prepared as an account of work sponsored by an agency of the United States Government. Neither the United States Government nor any agency thereof, nor any of their employees, makes any warranty, express or implied, or assumes any legal liability or responsibility for the accuracy, completeness, or usefulness of any information, apparatus, product, or process disclosed, or represents that its use would not infringe privately owned rights. Reference herein to any specific commercial product, process, or service by trade name, trademark, manufacturer, or otherwise, does not necessarily constitute or imply its endorsement, recommendation, or favoring by the United States Government or any agency thereof. The views and opinions of authors expressed herein do not necessarily state or reflect those of the United States Government or any agency thereof. 


\title{
ENGINE MATERIALS COMPATIBILITY WITH ALTERNATE FUELS
}

\section{ORNL/USCAR CRADA No. NFE-09-02242 FINAL REPORT}

\author{
PI: S. J. Pawel, co-PIs: J. K. Thomson and D. F. Wilson
}

Oak Ridge National Laboratory

One Bethel Valley Road

P.O. Box 2008, MS-6156

Oak Ridge, TN 37831-6156

USA

\section{ABSTRACT}

The compatibility of aluminum and aluminum alloys with synthetic fuel blends comprised of ethanol and reference fuel C (a 50/50 mix of toluene and iso-octane) was examined as a function of water content and temperature. Commercially pure wrought aluminum and several cast aluminum alloys were observed to be similarly susceptible to substantial corrosion in dry $(<50 \mathrm{ppm}$ water $)$ ethanol. Corrosion rates of all the aluminum materials examined were accelerated by increased temperature and ethanol content in the fuel mixture, but inhibited by increased water content. Pretreatments designed to stabilize passive films on aluminum increased the incubation time for onset of corrosion, suggesting film stability is a significant factor in the mechanism of corrosion.

\section{STATEMENT OF OBJECTIVES}

- Conduct a systematic assessment of aluminum corrosion in ethanol fuel

- Develop a mechanistic understanding of aluminum corrosion associated with increased ethanol content

- Identify cause(s) of potential galvanic corrosion associated with aluminum engine components

- Consistent with the corrosion mechanism, identify potential mitigation techniques

- Develop rapid test protocols to enable anticipation of potential problems in design stage

\section{BENEFITS TO THE FUNDING DOE OFFICE'S MISSION}

The ever increasing rate of fossil fuel consumption coupled with the desire for energy independence has influenced the United States to enact legislation to integrate renewable biofuels such as ethanol into the nation's fuel supply [1]. The U.S. Department of Energy (DOE) Office of Vehicle Technologies aims to facilitate this transition by removing technical hurdles and reducing cost barriers for emerging vehicle technologies. In alignment with this goal, the purpose of this investigation was to systematically examine the potential impact of changing fuel compositions on fuel compatibility with materials of interest for lightweight engine materials. The focus of the effort has been on aluminum and its alloys, primarily because of its low density and extensive use in automotive engines as a piston and head material for fuelefficient vehicles.

The addition of ethanol to gasoline is expected to be a source of concern for increased corrosion in two primary ways. Firstly, compared to gasoline, ethanol has significant affinity for water. Thus, additions of ethanol to transportation fuel invite the possibility of aqueous corrosion of all materials in contact with the fuel, including vehicle fuel systems and engines as well as the infrastructure for distributing and dispensing the fuel. Due to the hygroscopic nature of ethanol, absorbed water can separate from the 
organic fuel phase leading to a number of potential issues with fuel and containment corrosion [2]. Secondly, ethanol is orders of magnitude more electrically conductive than gasoline [3], so even modest additions of ethanol can contribute to corrosion possibilities in ethanol fuel blends, such as galvanic attack, that are not observed in ethanol-free gasoline. There are mixed reports suggesting variable degrees of corrosion susceptibility of aluminum in ethanol [4-11]. Inconsistencies exist possibly as a result of variations (intended or not) in critical exposure variables (principally water content) and/or incomplete interpretation of the results. An overview of several experiments demonstrating the unique vulnerabilities of aluminum alloys to corrosion in ethanol is presented.

\section{WORK PERFORMED BY ORNL}

The following is a summary of work performed at ORNL. Work performed by the USCAR partners was not released to ORNL for this report. The USCAR partners determined that certain information developed from the study is business sensitive. This report represents a summary of results extracted from documents that were approved by the partners for public release.

\subsection{Galvanic Corrosion Assessment}

Identically sized/shaped specimens $(1.3 \mathrm{~cm} \times 1.0 \mathrm{~cm} \times 0.8 \mathrm{~cm})$ of type 1100 aluminum, copper, cartridge brass, mild steel, and type 304 stainless steel were immersed individually and as couples with 1100 aluminum and one of the other materials in approximately $100 \mathrm{ml}$ of anhydrous ethanol (E100) purchased as a reagent grade chemical (Aldrich). In the case of couples, specimens of aluminum were connected to the other metal via a short $(\sim 1 \mathrm{~cm})$ section of threaded stainless steel rod, and the composite specimen was tightened until the ends of the different metals were in physical contact, and the surfaces under slight compression. The resistance across the couple was measured with a Fluke 287 multimeter and if not found to be less than $0.1 \mathrm{ohms}$, the contact was tightened and remeasured. The test vessel was a roundbottom glass flask fitted with a mantle-style heater and a water-cooled Allihn-style condenser. The opening at the top of the condenser incorporated a glass elbow filled with a molecular sieve material to prevent ingress of water vapor over the course of the experiment without actually sealing the heated vessel. The liquid temperature, which was initially at approximately $22^{\circ} \mathrm{C}$, was increased to $78^{\circ} \mathrm{C}$ (solution boiling point) and maintained constant for $24 \mathrm{~h}$. The water concentration of the E100 was measured before and after each experiment using Karl Fischer titration. Assessment of corrosion included visual appearance as well as dimensional changes and mass changes.

\subsection{Water Content, Temperature, and Ethanol Concentration Studies}

Tests were carried out in custom low volume $(\sim 100 \mathrm{ml})$ Teflon lined 316 stainless steel autoclaves. All test solutions were prepared from reagent grade chemicals and high-purity water $(18 \mathrm{M} \Omega-\mathrm{cm})$. Test fuel mixtures were based on the test-fuel standard specified in SAE J1681 [12], which was developed as a simulated gasoline for laboratory testing. The composition of the test fuel, often termed fuel ' $\mathrm{C}$ ' in the literature, is 50\% iso-octane and 50\% toluene. Synthetic purified ethanol, not bio-derived ethanol, was used to minimize variables which could be introduced based on the production and handling of fuel grade ethanol. [13] Aggressive formulations of the simulated fuel, containing the specified maximum limits of concentrations of potential contaminants such as acids and/or salts were also tested. Test solutions were mixed and loaded into the autoclaves along with the appropriate test specimen within an argon glove box

to exclude unintentional additions of water and oxygen. Test specimens included type 1100 aluminum, as well as specimens of 7 cast aluminum-silicon-magnesium-copper alloys representing a range of compositions of interest to the partners. One specimen per vessel per experiment was mounted into the bottom of each reaction vessel via a Teflon screw, and each experiment incorporated a nominal test solution volume of about $50 \mathrm{ml}$. After filling the reaction vessel inside the glove box, the mixture was shaken vigorously for $\sim 2$ minutes to ensure complete mixing, and a $\sim 1 \mathrm{ml}$ aliquot was removed for water 
content analysis by Karl Fischer titration. The reaction vessel was then sealed under argon and transferred to the test station where it was attached to a manifold fitted with a pressure relief valve (500 psi). The entire vessel and manifold were then purged with argon for $\sim 15$ minutes at room temperature. An additional set of experiments was conducted with an oxygen purge of the manifold and autoclave chamber to test the contribution, if any, of oxygen as a stand alone variable to the corrosion/passivation mechanism. Subsequently, equilibration at the desired temperature was accomplished with heat tapes for each vessel, and temperature was maintained constant $\left( \pm 1^{\circ} \mathrm{C}\right)$ for the duration of the experiment. Following the desired exposure, the vessels were allowed to quickly cool to room temperature, and a second aliquot $(\sim 1 \mathrm{ml})$ was removed for assessment of water content.

Assessment of corrosion was again primarily a yes/no evaluation based on visual changes on the specimen as well as pressure and temperature changes within the autoclave as the general performance boundary of aluminum in ethanol as a function of critical variables was sought. Where appropriate, mass change of the before and after test sample was also recorded.

\subsection{Pretreatment Studies}

A collection of seven commercial and experimental aluminum alloys was pretreated and tested for corrosion susceptibility in ethanol. The specimens underwent a pretreatment step based on the 'HFIR' surface treatment, named after a standard surface treatment given to aluminum fuel cladding at the High Flux Isotope Reactor at the Oak Ridge National Laboratory. This treatment includes immersion of the specimens in a room temperature solution of (by volume) $15 \%$ reagent grade nitric acid, $1 \%$ reagent grade hydrofluoric acid and 84\% demineralized water for 3 minutes. [14] The specimens are then rinsed in pure demineralized water and the passive film is allowed to heal itself into a continuous film in an additional volume of demineralized water for 3 minutes at $70^{\circ} \mathrm{C}$. For each test an untreated specimen was attached to the treated specimen with a Teflon screw to assure subsequent identical environmental exposure. The specimens were then tested for corrosion susceptibility in an identical setup as outlined in the galvanic assessment section above with the exception that the specimens remained in the test solution for a period of 72 hours rather than 24 .

\section{RESULTS and DISCUSSION}

\subsection{Galvanic Coupling}

Karl Fischer titration showed that the water content pre- and post-test was less than $100 \mathrm{ppm}$ in all cases. The corrosion results are summarized in Figures 1a and 2a, which pictorially indicates that uncoupled (stand-alone) aluminum specimens corroded to an essentially identical degree to the aluminum exposed in each galvanic couple. Further, the end of the aluminum specimen physically in contact with each coupled material revealed no more or less corrosion than the rest of the specimen, again suggesting no effect of electrochemical potential for these exposure conditions. In all cases, whether exposed as part of a couple with aluminum or as individual specimens, the other materials - copper, cartridge brass, mild steel, and 304 stainless steel - were found to be completely immune to corrosion under these conditions. The mass loss of aluminum material in each case was approximately $20 \pm 3 \%$. The corrosive attack on aluminum appears to initiate at discrete locations as rather intense general corrosion. These areas of intense corrosion have some similarities to pitting, but the corroded area spreads laterally at a similar rate to penetration, and ready overlap of corroded areas suggests no electrochemical protection of areas adjacent to those areas that are actively corroding. The experiment was repeated under similar test conditions with a $0.5 \%$ by weight water addition in E100. This resulted in a test fuel with a higher electrical conductivity, however, corrosion of the aluminum was suppressed due to the presence of the water. Results for this experiment are shown in Figures $1 b$ and $2 b$. 


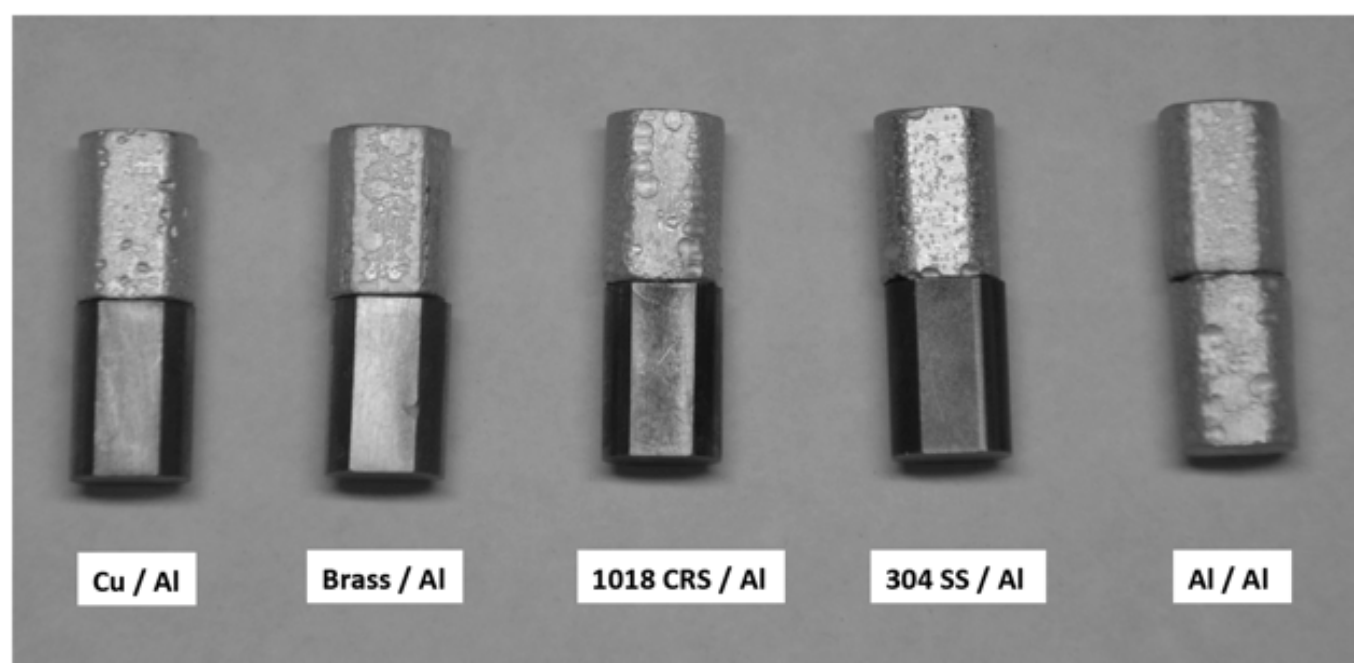

Figure 1a Galvanically coupled aluminum specimens and stand alone aluminum specimen exposed to anhydrous ethanol E100 for a period of 24 hours at $78^{\circ} \mathrm{C}$. In each couple, the aluminum sample is nearest to the top of the picture. All aluminum specimens exhibited similar corrosion.

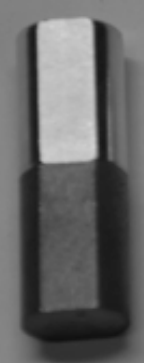

$\mathrm{Cu} / \mathrm{Al}$

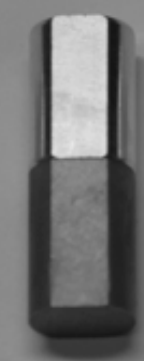

Brass/Al
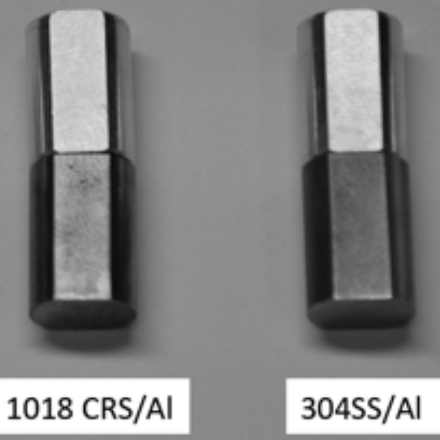

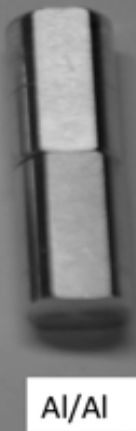

$\mathrm{Al} / \mathrm{Al}$

Figure 1b Galvanically coupled aluminum specimens and stand alone aluminum specimen exposed to ethanol E100 with $0.5 \%$ water for a period of 24 hours at $78^{\circ} \mathrm{C}$. In each couple, the aluminum sample is nearest to the top of the picture. No corrosion exhibited by aluminum specimens. 


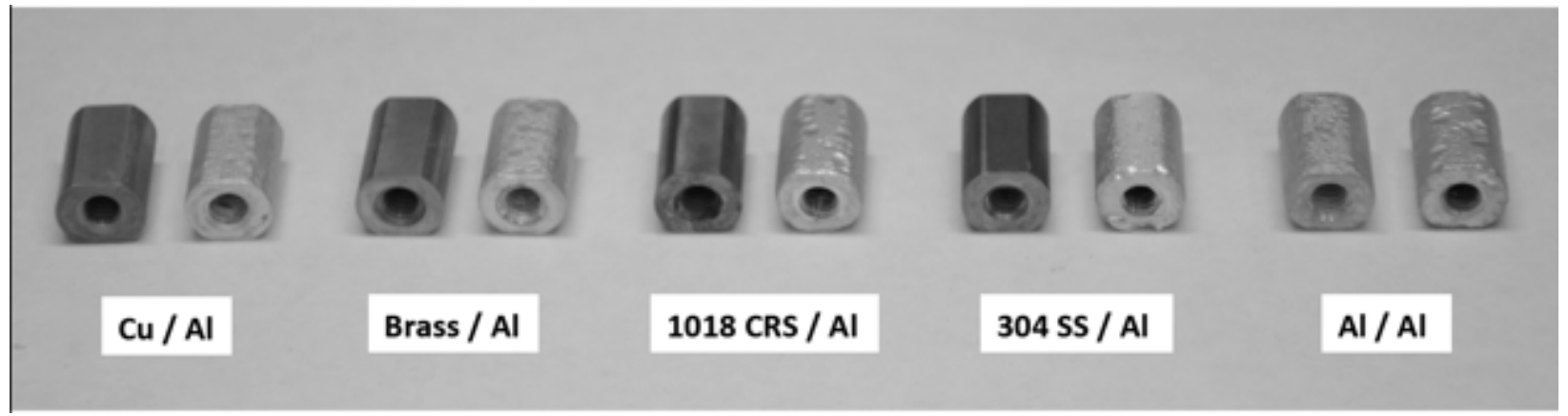

Figure 2a. Separated galvanic couples of Al 1100 with copper, cartridge brass, 1018 carbon steel and 304 stainless steel after exposure to anhydrous ethanol E100 for a period of 24 hours at $78^{\circ} \mathrm{C}$. No observed effect of galvanic coupling on the corrosion of aluminum.

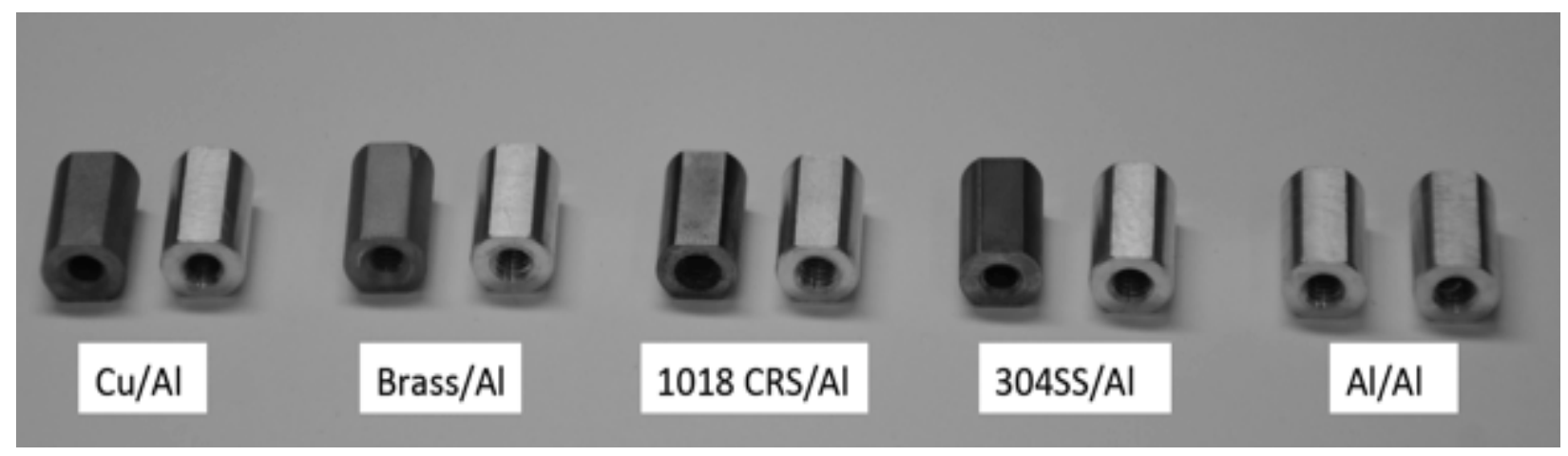

Figure 2b. Separated galvanic couples of Al 1100 with copper, cartridge brass, 1018 carbon steel and 304 stainless steel after exposure to ethanol E100 with $0.5 \%$ water for a period of 24 hours at $78^{\circ} \mathrm{C}$.

\subsection{Water Content and Temperature}

Results on the effect of water content and temperature on aluminum corrosion in ethanol are summarized in Table 1. In these tests, if the reaction initiates at all, it proceeds quite rapidly. The reaction is accompanied by a rapid increase in pressure and temperature as hydrogen gas is generated and the exothermic reaction accelerates. Gas samples were collected through a release valve into a Teflon sample bag and analyzed by a gas chromatograph/mass spectrometer (GC/MS). It was determined that hydrogen gas was the only gaseous reaction product. Samples of the post reaction liquid fuel were also analyzed by GC/MS and aside from the original fuel constituents, ethanol, toluene and iso-octane, no evidence was found of other compounds as reaction products. In the cases where the reaction initiates, the aluminum specimen is quickly consumed leaving a bright white powder interspersed with gray particles as solid corrosion product. For specimens tested in water concentrations below $0.1 \%$, post experiment Karl Fischer titrations demonstrated significantly decreased water concentration levels indicating that water is consumed in the reaction. The solid corrosion product was analyzed by scanning electron microscopy (SEM) and energy dispersive $\mathrm{x}$-ray spectroscopy (EDX), and $\mathrm{x}$-ray diffraction (XRD) and is thought to consist of a combination of amorphous aluminum oxide, aluminum hydroxide and aluminum oxyhydroxide. The experiments which were purged with oxygen rather than argon did not show a significant difference in reaction rates or reaction products. 
Table 1 Temperature and water concentration corrosion dependence of Al 1100 in E100 over a 24 hour time period. 'Yes' indicates rapid aluminum corrosion under designated conditions, whereas 'No' indicates no appreciable corrosion observed.

\begin{tabular}{cccccccc}
\hline Temperature $\left({ }^{\circ} \mathrm{C}\right)$ & \multicolumn{7}{c}{ Water Content } \\
& $\leq 50 p p m$ & $\mathbf{2 0 0} \mathbf{p p m}$ & $\mathbf{0 . 1 \%}$ & $\mathbf{0 . 5 \%}$ & $\mathbf{1 \%}$ & $\mathbf{5 \%}$ & $\mathbf{1 0 \%}$ \\
$\mathbf{2 0}$ & No & No & No & No & No & No & No \\
$\mathbf{4 0}$ & No & No & No & No & No & No & No \\
60 & No & No & No & No & No & No & No \\
80 & Yes & Yes & No & No & No & No & No \\
100 & Yes & Yes & Yes & No & No & No & No \\
120 & Yes & Yes & Yes & No & No & No & No \\
160 & Yes & Yes & Yes & Yes & Yes & No & No \\
200 & Yes & Yes & Yes & Yes & Yes & No & No \\
\hline
\end{tabular}

Due to rapid reaction progression under these conditions of susceptibility, an attempt to quantify corrosion rates would not be very useful. Therefore, test outcomes are reported in a binary 'yes' or 'no' format. In the case of a 'yes' result, for all intents and purposes, the aluminum sample was heavily attacked in a 24 hour time period. In the case of a 'no' result, no appreciable corrosion was observed. Experience in this laboratory has shown that if the reaction does not initiate in the first 24 hours, then it likely will not initiate on a much longer time scale. The data presented in Table 1 are specifically for type 1100 aluminum, but it should be noted that similar results were obtained for a series of several cast aluminum alloys as well. This observation indicates that the specific structure (wrought vs. cast, presence of largely un-attacked second-phase particles of much different composition than the base alloy) is not very important to the corrosion mechanism(s). Rather, it appears that aluminum is fundamentally incompatible with dry ethanol.

\subsection{Ethanol Concentration}

Increasing concentrations of ethanol in the anhydrous synthetic fuel blend resulted in increasingly rapid rates of corrosion. As depicted in Figure 3, aluminum is essentially immune to corrosion in E0 for the conditions of this experiment $\left(24 \mathrm{~h}\right.$, synthetic fuel at $\left.120^{\circ} \mathrm{C}\right)$. In E5, there are isolated, shallow indications of corrosion initiating on the surface. In E10, there are multiple, shallow areas of attack on the surface. In E15, the areas of attack are widespread, with increasingly large sections of the surface area being affected, and areas of corrosion are substantially overlapping. In E20, the entire surface layer has been attacked and aluminum is dissolving at a high and generally uniform rate. In addition to the results depicted here, examinations were also conducted in E50, E85 and E100, all of which resulted in even more extensive corrosion of the metallic specimen. Several cast aluminum alloys were also tested; all yielded generally similar results. Reaction products included hydrogen gas, an amorphous combination of aluminum oxide, aluminum hydroxide and aluminum oxy-hydroxide, as well as metallic second phase particles from the cast alloys, which were verified by SEM, EDX and XRD experiments. 


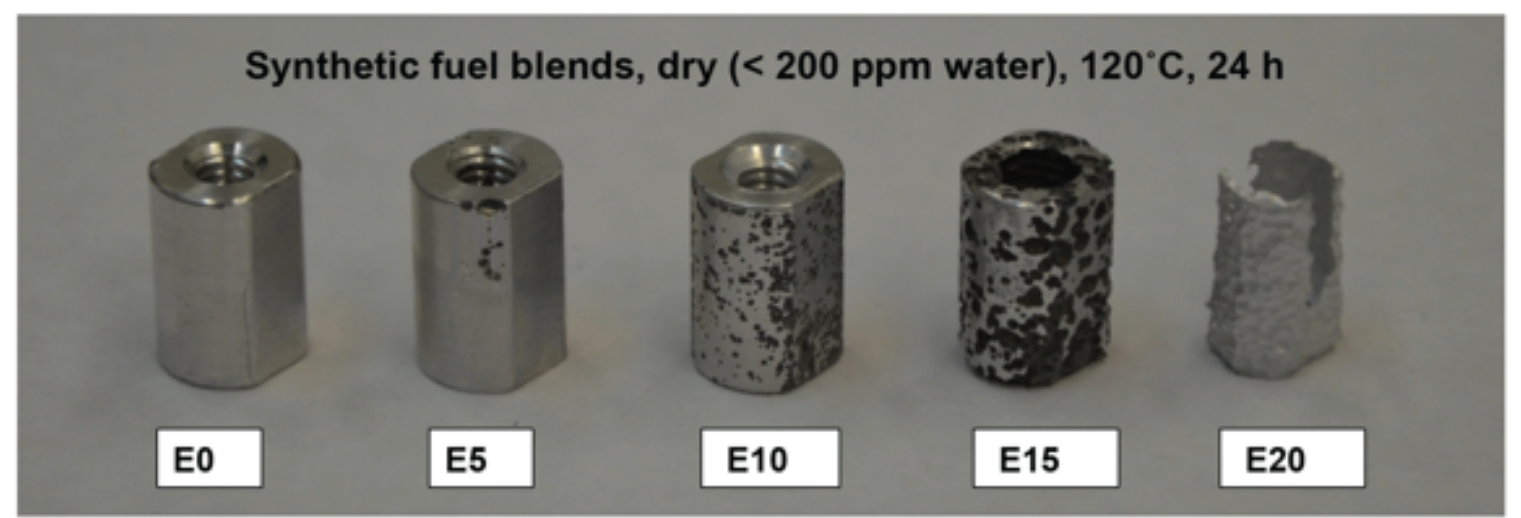

Figure 3. Corrosion of aluminum increased with increasing concentrations of dry ethanol synthetic fuel blend $\mathrm{C}$ at $120^{\circ} \mathrm{C}$ for a 24 hour time period.

\subsection{Pretreatment}

Representative results for three of the eight aluminum alloys receiving pretreatments and subsequently tested for corrosion susceptibility in ethanol are shown in Figure 4. In all cases, the treated alloys fared better than the untreated alloys in terms of surface damage and product accumulation. In general, the treated alloy exhibited less coverage of active corrosion for a fixed exposure period than did the untreated specimen. It should be noted that when the corrosion occurs on the treated alloys, it progresses in a similar fashion to the untreated alloys albeit with a longer incubation period. In general, treated alloys with low levels of copper, manganese and zinc exhibited higher levels of immunity from corrosive attack. In some cases, the reaction product, after saturating the limited solution volumes, would precipitate on an available surface, including that of the specimen, and the reaction rate would rapidly decrease due to a lack of available reaction sites. This phenomenon will be explored further in the next section.

\section{Untreated aluminum alloys}

\section{Treated aluminum alloys}

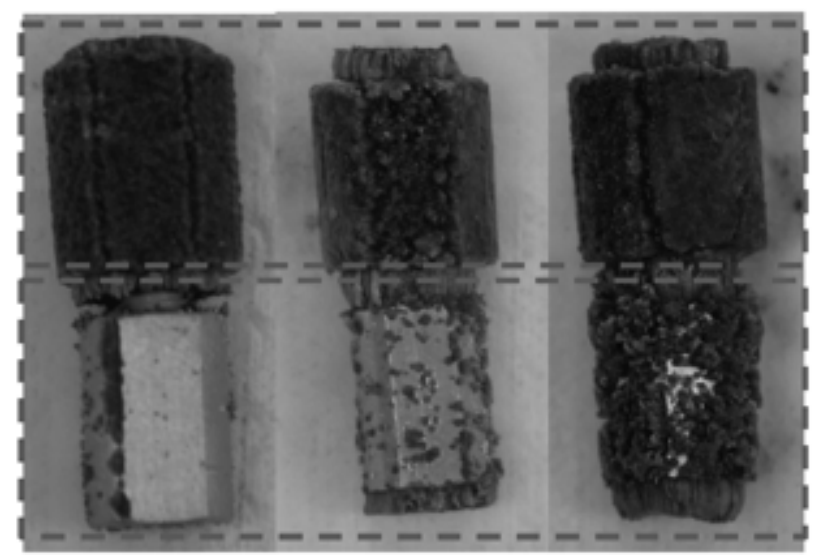

Figure 4. Aluminum alloys exhibiting corrosion in treated and untreated conditions. Pretreatment included immersion in dilute acid followed by boiling in purified water. Specimens were exposed to anhydrous $\mathrm{E} 100$ at $78^{\circ} \mathrm{C}$ for 72 hours. 


\subsection{Corrosion Mechanism}

According to the open literature on the subject, aluminum can corrode in alcohol in either a 'wet' pathway or a 'dry' pathway. [5-7] Depending on environmental conditions such as temperature, water concentration, exposure time, etc., one reaction may dominate over another, i.e., these reactions do not necessarily occur sequentially. The wet and dry families of reaction pathways are:

Wet Corrosion

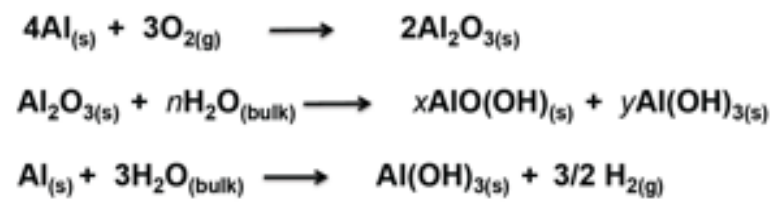

Dry Corrosion

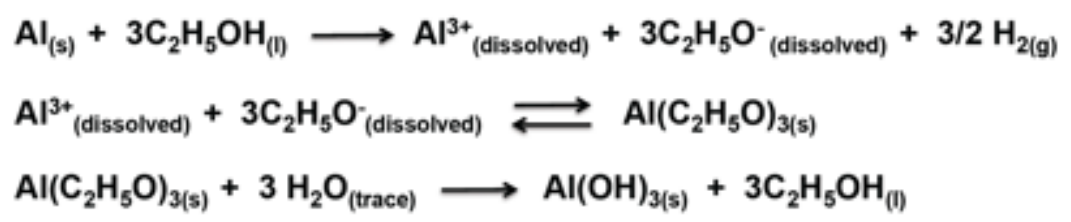

When metallic aluminum contacts either oxygen or water it will rapidly passivate forming a protective oxide layer on the aluminum surface. Under dry ethanol conditions, when the underlying metallic aluminum becomes exposed (through mechanical, chemical or other means), the metal cannot reform a passive layer due to the absence of oxygen and water, and corrosion proceeds unabated. As temperature increases, it is postulated that the mismatch of the thermal expansion coefficients for aluminum and aluminum oxide/hydroxide cause the outer passivated surface layer to crack, exposing metallic aluminum below the surface. Yoo et al., has suggested that the presence of anhydrous ethanol may also serve to dehydrate the passive film, leading to further cracking.[5] The corrosion reaction initiates at these discrete microcrack locations, gradually enlarging as metallic aluminum dissolves and hydrogen is generated. As the areas subject to corrosion increase, the reaction quickly accelerates leading to rapid general corrosion over the entire surface of the metal. Before the general corrosion can occur, there is a finite incubation time, typically on the order of a few hours, during which the passive layer becomes undermined possibly via coalescence of microcracks.

In aluminum alloys, second phase material does not dissolve as the reaction proceeds and remains generally unattacked in the growing areas of corrosion. The corroded surface turns black and becomes quite porous as aluminum continues to rapidly dissolve. Figure 5 demonstrates how the corrosion reaction proceeds under dry ethanol conditions for an aluminum alloy at $78^{\circ} \mathrm{C}$. Figure 6 shows high magnification SEM images of the reaction sequence for the specimens depicted in Figure 5. During the first 12 hours, the sample loses mass as aluminum attack spreads over the exposed surface. General corrosion spreads from the initiation point in all directions and corrosion proceeds until the entire surface becomes active. At some point beyond 12 hours, the specimen starts to regain mass as corrosion product, presumably aluminum ethylate, $\mathrm{Al}\left(\mathrm{C}_{2} \mathrm{H}_{5} \mathrm{O}\right)_{3}$, begins to precipitate on the metal surface. The precipitation of corrosion products appears to slow the reaction rate considerably. This result appears to be an effect of using a small volume of test fuel, which becomes saturated with dissolved species from the corrosion reaction. Under test conditions at higher temperatures or in larger solution volumes, this decrease in reaction rate was not observed. At temperatures above $100^{\circ} \mathrm{C}$, a very drastic and sudden spike in pressure $(\sim 500 \mathrm{psi})$ and temperature is observed after an incubation time typically between 3 to 5 hours. For the volume of solution used and size of the reaction vessel, this rapid pressure spike signals the reaction is 
nearing completion; in most cases the remaining metal is completely consumed over a period of less than a minute from the onset of the pressure spike. The rapid generation of hydrogen at the microcracks in the oxide layer serves to further disrupt the passive oxide layer. This sudden spike in temperature and pressure is likely the culmination of all the discrete initiation points coalescing into a single monolith of exposed metallic aluminum.

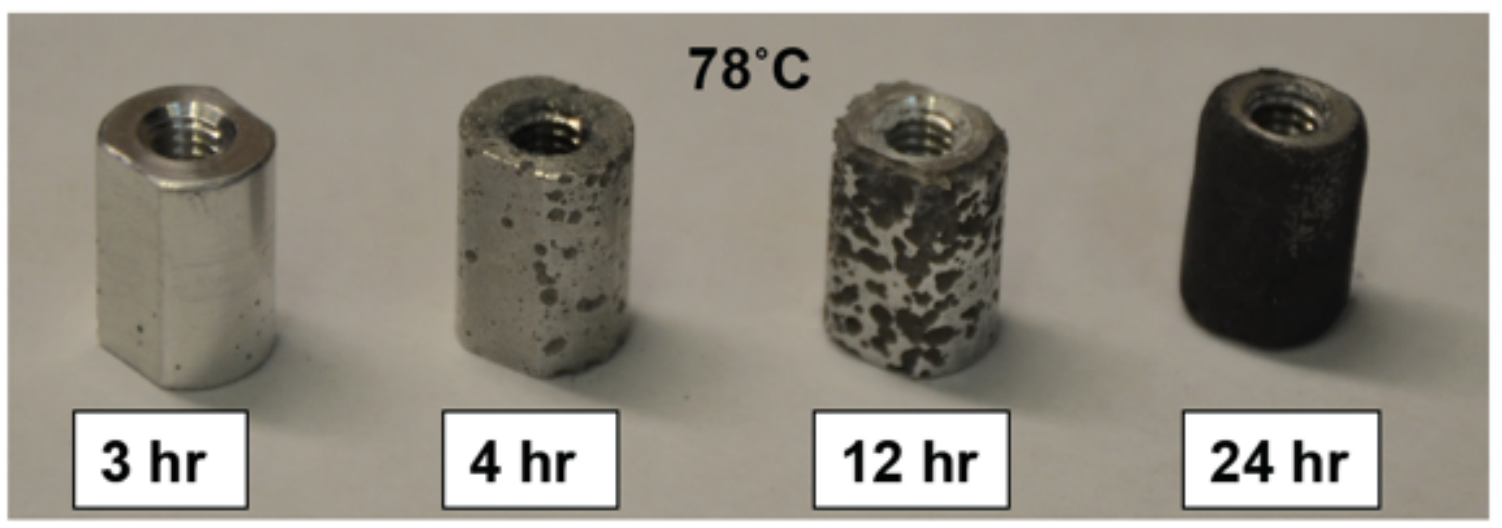

Figure 5. Aluminum alloy corrosion progression over a 24 hour time period. Specimens were exposed to anhydrous E100 at $78^{\circ} \mathrm{C}$.
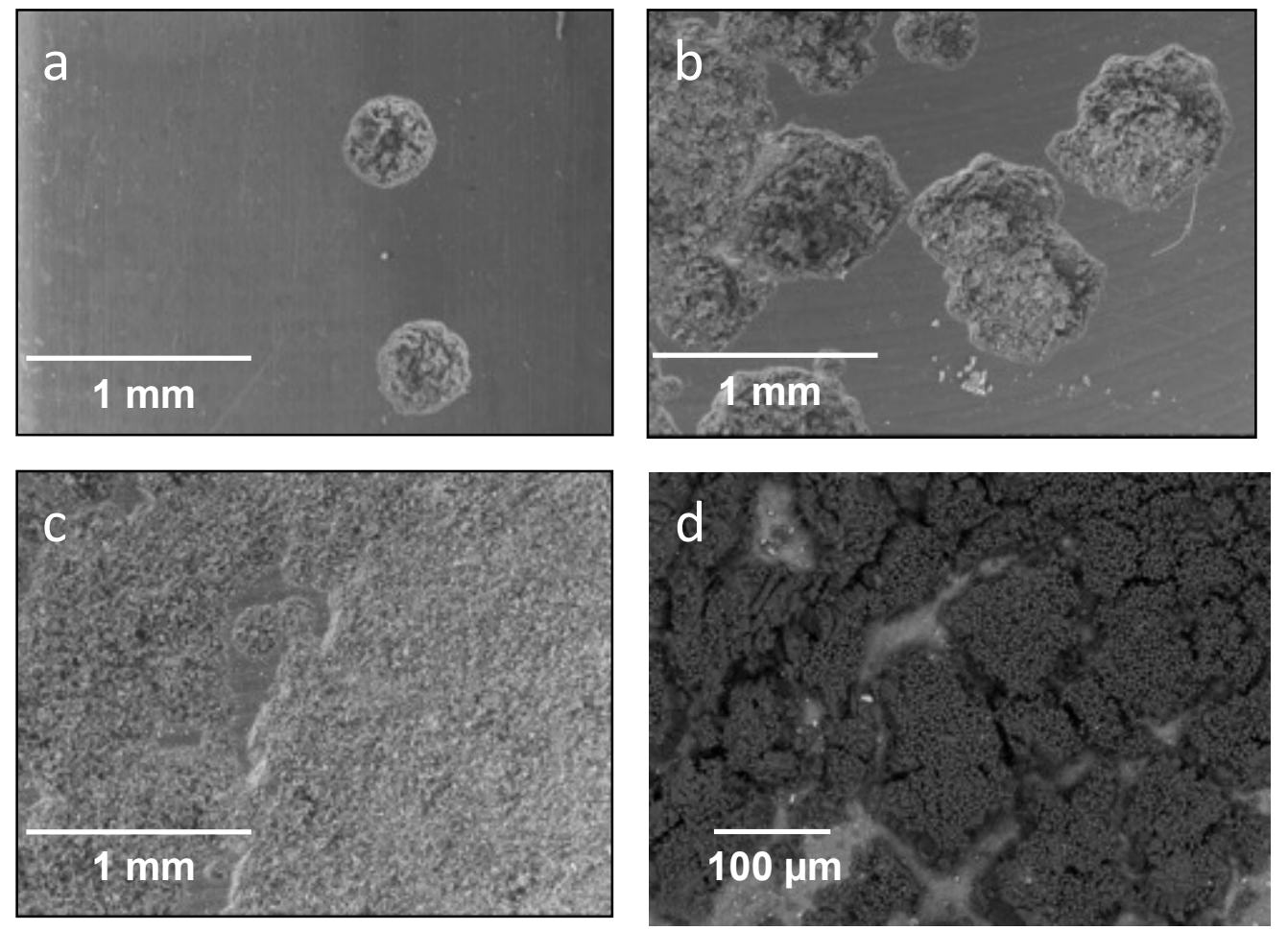

Figure 6. SEM images of aluminum alloy (corresponding to the specimens in Figure 5) exposed to anhydrous E100 at $78^{\circ} \mathrm{C}$ for time periods a) 3 hours, b) 4 hours, c) 12 hours, and d) 24 hours. 


\section{SUBJECT INVENTIONS}

Unfortunately, the timing and funding for this project were not of a sufficient duration and amount conducive to fully investigating a comprehensive mitigation strategy. It is anticipated that future inventions addressing aluminum corrosion in ethanol would likely include: novel aluminum alloy design, surface pre-treatments, novel coating technologies and/or fuel additive packages designed to inhibit corrosion.

\section{COMMERCIALIZATION POSSIBILITIES}

None at the present time

\section{PLANS FOR FUTURE COLLABORATION}

The USCAR Partners signaled a preference to conduct future research in their own internal research facilities where they can more tightly protect sensitive information. ORNL would welcome future collaborations with other stakeholders interested in corrosion research involving ethanol containing fuels.

\section{CONCLUSIONS}

On-going investigation of aluminum compatibility with ethanol containing fuels has shown that aluminum and dry ethanol are fundamentally incompatible. It has been demonstrated that aluminum is most vulnerable to extensive corrosion in ethanol environments having trace water concentrations, at elevated temperature, and after exposure times on the order of hours. Fortunately, most real-world aluminum ethanol contact does not happen under conditions that allow for "dry" corrosion in ethanol to occur. The present study was carried out under tightly controlled laboratory conditions, using reagent grade simulated fuel. Therefore, the effect of impurities from the field is currently unknown and worthy of future study. Observations of aluminum/ethanol corrosion from the field appear to be isolated to areas where uncombusted fuel is allowed to pool and stagnate. Aluminum alloy engines running on E85 have been in operation for over 10 years and have shown very minimal susceptibility to this type of corrosion in ethanol. What has been learned in the present study can be summarized as follows: on exposure to dry ethanol, aluminum exhibits a finite incubation period during which time the passive oxide layer is penetrated, exposing the reactive base metal to ethanol with which it rapidly reacts. The reactivity rate is strongly dependent on the concentration of water in the ethanol fuel. At water concentrations below 1\%, aluminum is susceptible to rapid rates of corrosion at temperatures above $80^{\circ} \mathrm{C}$. High levels of water suppress aluminum reactivity with ethanol, by allowing the passive aluminum oxide or hydrated aluminum oxide layer to remain protective or to reform if penetrated. Viable routes for investigation to mitigate this reactivity/corrosion problem include: (a) the development of an aluminum alloy composition that allows for a more tenacious, less defective, passive oxide layer and/or a more rapid reforming of a protective layer, especially at lower water levels; and (b) development of an additive package that is compatible with engine performance and fuel economy that decreases aluminum reactivity in the presence of ethanol. The former approach, alloy development, would start with modeling of the alloy with computational thermodynamics to reduce the design space. The latter, an additive package, might begin with the inclusion of known aluminum passivating agents, or at the very least, setting a lower bound for acceptable water concentration in ethanol containing fuels. 


\section{REFERENCES}

[1] The Energy Policy Act of 2005 (EPAct 2005, P.L. 110-58), The Energy Independence and Security Act of 2007 (P.L. 110-140, H.R. 6) See http://www.ethanol.org/index.php?id=78.

[2] Vargel C. Corrosion of aluminum. Oxford: Elsevier Ltd: 2004.

[3] Kirk DW. Conductivity of gasoline ethanol water mixtures. Fuel 62 (1983) 1512-3.

[4] Niven, R.K. Ethanol in gasoline: environmental impacts and sustainability review article. Renewable and Sustainable Energy Reviews 9 (2005) 535-555.

[5] Yoo, Y.H.; Park, I.J.; Kim, J.G.; Kwak, D.H.; Ji, W.S. Corrosion characteristics of aluminum alloy in bio-ethanol blended gasoline fuel: Part 1. The corrosion properties of aluminum alloy in high temperature fuels. Fuel 90 (2011) 1208-1214.

[6] Scholz M, Ellermeier J. Corrosion behavior of different aluminum alloys in fuels containing ethanol under increased temperatures. Mat -wiss U Werkstofftech 2006:37: 842-51.

[7] Kruger, L.; Tuchscheerer, F.; Mandel, M.; Muller, S.; Liebsch, S., Corrosion behavior of aluminum alloys in ethanol fuels, J Mater Sci, (2012) 47: 2798-2806.

[8] Pawel, S.J.; Kass, M.D.; Janke, C.J.; Preliminary Compatibility Assessment of metallic dispenser materials for service in ethanol fuel blends. ORNL/TM-2009/286.

[9] Nie, X.; Li, X; Northwood, D. Corrosion Behavior of metallic materials in ethanol-gasoline alternative fuels. Materials Science Forum Vols. 546-549 (2007) 1093-1100.

[10] Kane, R.D.; Maldonado, J.G. Stress Corrosion Cracking in Fuel Ethanol: a Newly Recognized Phenomenon. NACE Corrosion 2004 Paper No. 04543.

[11] Nakaguchi, G.M.; Keller, J.L.; Wiseman, E.L., Ethanol Fuel Modification for Highway Vehicle Use Final Report. Union Oil Company of California, ALO-3683-T1, 1979.

[12] The Engineering Society for Advancing Mobility Land Sea Air and Space, "Gasoline, Alcohol, and Diesel Fuel Surrogates for Materials Testing”, SAE J1681 Revised Jan 2000.

[13] Harrigan, M.J.; Banda, A.; Bonazza, B.; Graham, P.; Slimp, B. A Rational Approach to Qualifying Materials for Use in Fuel Systems. SAE 2000-01-2013.

[14] Pawel, S.J.; Manneschmidt, E.T., Journal of Nuclear Materials 318 (2003) 355-364.

\section{PUBLICATIONS}

A manuscript entitled 'Susceptibility of Aluminum Alloys to Corrosion in Simulated Fuel Blends Containing Ethanol' has been accepted by Fuel and will be published in 2013. 